\title{
Las voces de los jóvenes de una escuela secundaria técnica tradicionalmente masculina acerca de la educación y el trabajo
}

\section{The voices of the young people of a traditionally male technical secondary school on education and work}

\author{
ANA MARÍA D’ANDREA* \\ MARÍA PAULA BUONTEMPO** \\ José Antonio PozZER ${ }^{* *}$
}

El objetivo de este artículo es comprender las reflexiones de los jóvenes que cursan la especialidad Madera y Muebles en una escuela técnica de la provincia de Corrientes (Argentina) acerca de la educación y el trabajo y cómo estos temas son atravesados por la categoría género. Para recuperar sus voces, aplicamos encuestas y organizamos grupos focales con varones y mujeres, por separado. Observamos diferencias en las expectativas laborales y vivencias formativas entre estudiantes de ambos sexos, en las que se reproducen estereotipos de género. Concluimos que uno de los principales desafíos es visibilizar las problemáticas de las mujeres en las escuelas técnicas y avanzar en la inclusión del enfoque de género en las políticas públicas.

The objective of this article is to understand the reflections that young people studying the specialty Wood and Furniture in a technical school in the province of Corrientes (Argentina) make about education and work and how these issues are crossed by the gender category. In order to recover their voices, surveys and focus groups are conducted with men and women, separately. There are differences in work expectations and training experiences between students of both sexes, where gender stereotypes are reproduced. It is clear from the study that one of the main challenges is to make visible the problems that women face in technical schools and to advance in the inclusion of the gender approach in public policies.
Palabras clave: educación de la mujer, enseñanza técnica, igualdad de oportunidades

\section{Keywords:}

women's
education,
technical
education,
equal
opportunity

Recibido: 23 de diciembre de 2018. | Aceptado para su publicación: 28 de julio de 2019. Recuperado de: https://sinectica.iteso.mx/index.php/SINECTICA/article/view/935 doi: 10.31391/S2007-7033(2019)0053-005

Sección: Investigaciones temáticas

\footnotetext{
* Doctora en Antropología Social. Docente-investigadora de la Universidad Nacional del Nordeste (Argentina). Responsable del Área de Investigación del Ministerio de Educación de la Provincia de Corrientes (Argentina). Áreas de investigación: políticas educativas, formación para el trabajo, educación de jóvenes y adulto. Correo electrónico: anadandrea@gmail.com/http://orcid.org/0000-0001-9352-7255.

${ }^{* *}$ Doctora en Antropología Social. Docente-investigadora de la Universidad Nacional del Nordeste (Argentina). Integrante del Área de Investigación del Ministerio de Educación de la Provincia de Corrientes (Argentina). Áreas de investigación: políticas educativas, educación de jóvenes y adultos, educación digital. Correo electrónico: buontempop@gmail.com

*** Doctorando en Ciencias Sociales. Docente-investigadora de la Universidad Nacional del Nordeste (Argentina). Áreas de investigación: trayectorias educativas y laborales de jóvenes, formación para el trabajo. Correo electrónico: japozzer@yahoo.com.ar.
} 


\section{INTRODUCCIÓN}

ctualmente, en la Argentina, las mujeres acceden a niveles educativos más A altos que los hombres, pero eso no se ha traducido en un aprovechamiento en - términos de una inserción laboral y remuneración equitativas. Con un índice de masculinidad (el número de varones por cada 100 mujeres) del 94.8\%, según el último censo, el número de mujeres supera al de los varones en el país (Argentina, INDEC, 2011). Esto significa que la consideración de la igualdad de oportunidades laborales implica no solo un reconocimiento a los derechos de las mujeres, sino un aporte que estas pueden hacer al sistema productivo.

Si nos detenemos en la modalidad que intenta articular educación y trabajo, como es la educación técnico-profesional (ETP), según información del Instituto Nacional de Educación Técnica (INET), la matrícula femenina empezó a crecer en las escuelas técnicas. Sin embargo, permanece una proporción mayor de estudiantes varones que de mujeres. Las alumnas matriculadas en ETP (formación profesional, secundario técnico y superior técnico) representan el $43 \%$ del total. Este porcentaje todavía es menor en la escuela secundaria técnica (EST), ya que solo el 32.5\% son mujeres (Argentina, INET, 2018). No obstante, la proporción de mujeres y varones varía de acuerdo con la especialidad, siguiendo la lógica tradicional de la división sexual del trabajo: las mujeres son minoritarias en Mecánica (12\%), Electrónica y Energía (12\%) y Construcción (28\%), mientras que son mayoritarias en Administración (62,5\%) y en otras de Servicios (71.6\%) (Seoane, 2013). A cuatro años de haber terminado la secundaria, las mujeres continúan estudiando en un $70 \%$ de los casos, casi 15 puntos por encima de sus pares varones. Sin embargo, son menos quienes eligen seguir con alguna carrera técnica (55\% de mujeres y 77\% de varones) (INET, 2018).

En este trabajo nos interesa profundizar en aquellas especialidades que, tradicionalmente, fueron "masculinas" y en las que, poco a poco, fueron ingresando cada vez más mujeres. Consideramos la especialidad Industrialización de la Madera y el Mueble de una escuela técnica de Paso de los Libres (Corrientes) que, además, tiene otras dos especialidades no tradicionales para mujeres (Construcciones y Electromecánica). Nos focalizamos en esta especialidad porque en la provincia el sector productivo más dinámico es el forestal y, en los últimos años, las políticas educativas empezaron a acompañar su desarrollo (D’Andrea, 2018; D’Andrea y Buontempo, 2018).

Nos preguntamos ¿qué piensan los jóvenes que asisten a una especialidad considerada tradicionalmente masculina acerca de la educación y el trabajo? ¿Se perciben diferencias entre varones y mujeres respecto a las motivaciones que inciden en la elección de la escuela y la especialidad, a las experiencias en la institución y a las expectativas educativas y laborales? Si las hubiera, ¿cuáles son?

Este artículo forma parte de un estudio mayor cuyo objetivo es analizar cómo se producen y reproducen a nivel institucional las desigualdades entre varones y mujeres, e identificar las acciones tendentes a promover la igualdad de género en las EST y los centros de formación profesional.

En este trabajo presentamos los hallazgos obtenidos de las encuestas y los grupos focales realizados con estudiantes de una EST del interior de la provincia de Corrientes. El objetivo de este artículo es comprender, desde las voces de los jóvenes que asisten a una especialidad de educación técnica considerada tradicionalmente 
masculina, cómo perciben el ingreso, la formación recibida y las expectativas educativas y laborales, todo ello atravesado por el género.

En términos de género, existe abundante evidencia empírica acerca de la segregación ocupacional del mercado de trabajo, como también de las desigualdades basadas en género a nivel institucional en los centros educativos y en las prácticas y los discursos de varones y mujeres, en los que se producen y reproducen "códigos de género" (Subirats, 1986).

Aportar reflexiones desde los propios jóvenes resulta fundamental para analizar los mecanismos institucionales a través de los cuales las desigualdades se cristalizan en mandatos y destinos socialmente esperados para varones y mujeres. Con esto también pretendemos contribuir a los estudios vinculados a los Objetivos de Desarrollo Sostenible de la Organización de las Naciones Unidas (ONU), que plantean la transversalización de la perspectiva de género como herramienta metodológica para valorar políticas y legislaciones desde el punto de vista de sus implicaciones sobre varones y mujeres (ONU, 2015).

\section{REFERENTES TEÓRICOS CONCEPTUALES}

El género es el conjunto de ideas, representaciones, prácticas y prescripciones sociales que una cultura desarrolla desde la diferencia anatómica entre los sexos para simbolizar y construir socialmente lo que es propio de los hombres (lo masculino) y de las mujeres (lo femenino) (Lamas, 2000).

La incorporación de la perspectiva de género en las políticas es el resultado de debates en torno a la afirmación de la igualdad de oportunidades y condiciones laborales, educativas y sociales entre mujeres y varones. El recorrido histórico sobre esos debates permite reconocer, en palabras de Millenaar (2018), un profundo cambio de paradigma en las últimas décadas acerca de la ampliación de los derechos de las mujeres. La perspectiva de género nos permite comprender las diferentes posiciones que adultos y jóvenes, mujeres y varones ocupan en el espacio escolar, así como las disposiciones que muestran en relación con la escuela. Las dinámicas de género se nos presentan como interaccionales, en las que predominan jerarquías y asimetrías.

Como plantea Scott (1996), el género resulta una categoría analítica "útil" para revisar y abordar el modo en que se producen, utilizan y cambian los significados de los cuerpos sexuados, y descarta que las nociones de masculino y femenino sean fijas e invariables. La perspectiva de género, como señala Millenaar (2018),

\footnotetext{
... busca reconocer los vínculos que se producen entre normas, mandatos y relaciones de poder y saber, cristalizadas en instituciones, leyes, reglas y creencias, y las subjetividades y cuerpos, porque en esas relaciones se conforman significados en torno de la diferencia sexual. Entonces, para ubicar esta definición en la problemática de las relaciones juventud, educación y trabajo, podemos pensar en el orden simbólico de género que organiza y da forma a los procesos educativos y da entrada al mundo del trabajo (y que delinea recorridos diferentes para varones y mujeres, por ejemplo, a través de mandatos, expectativas sociales, pero también habilitaciones y restricciones específicas en términos de carreras educativas y de los empleos en el mercado de trabajo) y en el modo en que las subjetividades se vinculan (adhiriendo, resistiendo, reproduciendo o subvirtiendo) a dicha organización estructural y simbólica (p. 3).
} 
Los aportes de la teoría de género ayudan a reconocer el modo en que los "códigos de género" (Subirats, 1986), presentes en las instituciones educativas, inciden en cómo, alumnos y alumnas, llegan a una identificación personal en términos de varón y mujer. Esto se vincula a lo que Segato (2003) postula como "gramática de género". La misma se traduce en un mapa cognitivo con el que los sujetos operan. La interpretación de ese mapa es siempre una instancia individual y puede ser bastante aleatoria y accidentada. Así, no es posible pensar las identidades de género como algo acabado, sino como un proceso en constante transformación.

En su mayoría, las investigaciones relevadas sostienen que la oferta educativa de la ETP se encuentra en sí misma segregada en términos de género. Las especialidades que se ofrecen, tanto en la EST como en la formación profesional, de algún modo reflejan el patrón de división sexual del trabajo existente en el mercado laboral. A pesar de que es posible reconocer una afianzada reproducción de "códigos de género" hegemónicos, también es necesario resaltar la heterogeneidad de experiencias biográficas y subjetivas de quienes llegan a las aulas.

En una investigación desarrollada durante 2004 en cinco países europeos (Alemania, Finlandia, Gran Bretaña, Grecia y Portugal), Evans (2006) indagó el impacto de la segregación de género del mercado laboral en la ETP considerando tres especialidades: cuidado de niño/as, electricidad y cocina/servicio de camareros. El estudio mostró que varones y mujeres suelen elegir ocupaciones típicas de acuerdo con el género, porque se ven atravesados por expectativas sociales y, por ende, son entrenados en competencias también típicas conforme a su género. Estas, por lo tanto, del mismo modo se encuentran generizadas de manera acorde con la segregación de género que presentan las ocupaciones. En ese interjuego entre expectativas sociales, división sexual del trabajo y competencias, la segregación de géneros se reconstruye y actualiza una y otra vez.

Al estudiar las tres especialidades, observamos que en ellas las competencias más valoradas son las ligadas al género predominante en tal ocupación; por ejemplo, en el caso de cuidado de niños, las competencias más significativas refieren a la ternura, la sensibilidad y la paciencia, que son todos atributos asociados a los rasgos típicos de la feminidad. En la formación en cocina, más neutral en términos de género, advertimos diferencias de acuerdo con las tareas más afines para uno y otro género: las duras y pesadas para los varones y las livianas, "decorativas" y estéticas para ellas.

Las investigaciones muestran que las mujeres, cuando transitan por dispositivos orientados a actividades laborales estereotipadamente masculinas, deben sobrellevar permanentes obstáculos durante la experiencia educativa, y luego en el momento de la inserción laboral, en cuanto a la especialidad elegida, se privilegia la incorporación de nuevos ingresantes varones, a pesar de que tienen el mismo título y la misma formación. En este sentido, no siempre resulta en una discriminación explícita, pero sí contribuye a desalentar la inclusión en esas especialidades (Millenaar, 2017).

No solo el modo en que se plantea la oferta educativa manifiesta segregación de género. Algunos estudios relevados muestran también que los procesos de ingreso a la modalidad de ETP conllevan estrategias de reclutamiento y selección diferenciada. Bloj (2017) explica que el ingreso de mujeres a la educación técnica se encuentra permeado por un proceso de selección poco claro que va segregando a las jóvenes con menor capital cultural en las escuelas con menores recursos. Esta 
dinámica perjudica el desempeño de muchas estudiantes y reproduce esquemas de representación en los que el recurso a la meritocracia y al esfuerzo individual oculta mecanismos decisivos para la reproducción de las desigualdades de clase y género.

En el caso de la EST, este proceso no se digita racionalmente en las instituciones, sino que deviene como un proceso que es consecuencia de las lógicas propias de la formación. Las exigencias, horarios de taller, el entorno mayoritario de varones y las prácticas educativas son algunos elementos que van desestimulando a las mujeres respecto de la continuidad en la institución, sobre todo aquellas con menores capitales económicos y culturales.

En otros términos, si bien la escuela es la proveedora de capital cultural (Bourdieu, 1998), las EST solo ofrecen educación de nivel secundario. Esto implica que la EST hace un proceso de "destilación fraccionada", en palabras de Dubet y Martuccelli (1998), diferente de lo que ocurría décadas atrás cuando la selección operaba con mayor atención en el ingreso.

El carácter sexista y reproductor de las desigualdades de género que tiene la ETP, según venimos planteando, hunde sus raíces en la historia de la modalidad (León, 2009; Seoane 2017). León (2009) y Milleneaar (2018) plantean que hace tiempo, en las escuelas industriales, se obstaculizaba el ingreso de mujeres. Esto no se debía a ningún impedimento formal, sino a un imaginario que operaba limitando su acceso: por razones edilicias (no contar con baños para mujeres), por razones de convivencia (no es apropiado que las chicas estén rodeadas de varones) o de utilidad (de qué les sirven a las mujeres los aprendizajes técnicos y tecnológicos). Si bien este imaginario se fue modificando en los últimos años y la incorporación de mujeres a la escuela técnica es un hecho expandido, hoy persisten prácticas discriminatorias dentro de esos espacios educativos que obligan a las alumnas a ponerse a prueba permanentemente en cuanto a sus capacidades y también su tolerancia.

Coincidimos con Bloj (2017) en que las mujeres en las escuelas técnicas aparecen para ciertos grupos como "la otredad", ya que sus elecciones educativas en esta modalidad son concebidas como rarezas en algunas especialidades, sobre todo aquellas consideradas masculinas.

Seoane (2013) sostiene que la escuela, al igual que las instituciones que forman el entramado social, socializa a las nuevas generaciones en un determinado sistema sexo/género en el que, la mayoría de las veces, se reproducen (descuidadamente, o no) desigualdades y visiones estereotipadas respecto de lo femenino y lo masculino y, en otras (desde nuestro punto de vista, las menos), se habilitan espacios para debatir y desnaturalizar los mandatos sociales que recaen sobre mujeres y varones.

Dicha autora también apuesta a la hipótesis de que la matriz fundacional de la escuela técnica funciona como contenido fundamental de la socialización escolar no solo de las mujeres, sino también de los varones. Esta matriz, en ocasiones, estigmatiza y discrimina a los estudiantes por su condición social, juvenil, sexual y étnica, y también construye un sentido de pertenencia que los diferencia de aquellos que asisten a otras escuelas (Seoane, 2012). Así, el sexismo y el androcentrismo son formas de discriminación y como práctica están presentes en las instituciones educativas y son parte de la socialización de las nuevas generaciones (Morgade, 2001). Según el Diccionario de estudios de género y feminismo, el sexismo se define como "el 
mecanismo por el que se concede privilegio a un sexo en detrimento de otro". Para que se produzca, es necesario lo que se define como androcentrismo: "La concesión del privilegio al punto de vista del hombre" (Gamba, 2007, p. 293).

Los Objetivos de Desarrollo Sostenible plantean la transversalización de la perspectiva de género propuesta por la ONU como herramienta metodológica para valorar políticas y legislaciones desde el punto de vista de sus implicaciones sobre varones y mujeres. En el plano de la ETP, se ha propuesto el desarrollo de estrategias para "desnaturalizar" las opciones profesionales por género, y la revisión de mandatos impuestos por la cultura (Silveira, 2001 y 2011). Investigaciones muestran que, en algunos casos, la ETP ha incorporado estrategias que reconocen la perspectiva de género, como la inclusión de mujeres en ámbitos donde han estado subrepresentadas, históricamente, a través de la fijación de "cuotas" de participación, y la incorporación de acciones de deconstrucción de la desigualdad de género, mediante una revisión reflexiva y crítica de la división sexual del trabajo (Millenaar, 2016) y la sanción de la Ley Nacional de Educación Sexual Integral, que supone un avance en materia de incorporación de contenidos de perspectiva de género en la escuela secundaria, aunque existen resistencias para su plena implementación (Lavigne, 2009).

Esto es complementado con otros estudios sobre las desigualdades de género en la ETP, que indican ejes de indagación fundamentales para nuestro estudio: se señala que la composición de género del cuerpo docente y la división sexual del trabajo académico son importantes en la reproducción (o cuestionamiento) de estereotipos (Conde, 2013) y que las perspectivas de los docentes en torno a las diferencias de género influyen en el currículo oculto (Jackson, 2001), aspectos que, según la perspectivas de género, hay que revisar (Silveira, 2011).

\section{LA EDUCACIÓN TÉCNICA ARGENTINA}

La educación técnica argentina se gestó en el último cuarto del siglo XIX y se consolidó en el primer cuarto del siglo XX. En un principio, tenía dos tipos de destinatarios: las escuelas industriales eran para varones y las profesionales para mujeres. Ambas estaban orientadas a quienes deseaban tener una salida laboral inmediata y no querían o no podían continuar estudios superiores. Con el tiempo, los objetivos primigenios fueron variando, así como los perfiles de las escuelas técnicas. En 1964 se unificaron las escuelas industriales con las escuelas fábricas para varones y las profesionales y de fábrica para mujeres en una sola denominación: Escuela Nacional de Educación Técnica (Sobrevila, 1995).

Las primeras escuelas profesionales destinadas a mujeres tenían por función prepararlas para cumplir un rol fundamental como protectoras del hogar (Seoane, 2013). En los años setenta del siglo XX, comenzaban a ver disminuir su matrícula en virtud de la incorporación de las mujeres al mercado de trabajo y de nuevas expectativas y demandas. Por eso, muchas de ellas adoptaron la especialidad Administración de Empresas para prepararse en ocupaciones de oficina del sector terciario de la economía (Gallart, Miranda, Peirano y Sevilla, 2003).

En 2005, la Ley de Educación Técnico-Profesional destacaba, en el artículo 40, que "se ejecutará una línea de acción para promover la incorporación de mujeres como 
alumnas en la educación técnico-profesional.... En este marco, actualmente se observa un crecimiento de la matrícula femenina como señalamos en la introducción, aunque se estancó en el 30\% de mujeres en los últimos años. Por otra parte, en 2018 se creó la Comisión de Género en el marco de la ETP. Este organismo tiene como propósito incorporar la perspectiva de género e incrementar el número de mujeres en la modalidad en lo que se refiere al alumnado, equipo docente y directivo.

\section{Metodología}

El objetivo de este artículo es comprender las reflexiones de los jóvenes, varones y mujeres, acerca de la educación y el trabajo y cómo estos temas están atravesados por la categoría género. El trabajo de campo lo llevamos a cabo en septiembre de 2017. Nos interesa profundizar en aquellas especialidades que, tradicionalmente, fueron "masculinas" y en las que, poco a poco, fueron ingresando cada vez más mujeres. En específico, consideramos la especialidad Madera y Muebles de una escuela técnica de Paso de los Libres (Corrientes).

Para recuperar las voces de los cursantes, aplicamos una encuesta y conformamos grupos focales. En la encuesta, los temas estuvieron organizados en los siguientes módulos: formación técnica, formación técnica y trabajo, situación laboral, tareas en el hogar, características sociodemográficas. Y en los grupos focales, los módulos fueron: significados asociados a la especialidad, valoración de la formación recibida, diferenciación por género, visión del mercado de trabajo y expectativas futuras. Además, apelamos a la utilización de afiches para promover la discusión sobre cómo es ser varón o cómo es ser mujer en esa especialidad y en esa EST.

La encuesta fue respondida por todos los cursantes de la especialidad (42) y participaron en los grupos focales las seis mujeres presentes y los seis estudiantes varones del último año. No consideramos a quienes estaban ausentes el día del trabajo de campo (8). Acordamos mantener el anonimato de las personas participantes. La encuesta fue procesada con el SPSS (Statistical Package for the Social Sciences). Los intercambios de los grupos focales fueron grabados y luego transcritos para ser procesados con el software Atlas.ti.

Los resultados fueron organizados en función de las siguientes categorías: características personales y familiares, experiencias respecto a las dinámicas institucionales y de género, motivos de la elección de la escuela y la especialidad, valoración de la formación recibida y expectativas educativas y laborales. Para ello, privilegiamos los resultados de los grupos focales y solo seleccionamos los resultados del cuestionario que permitían comprender mejor las categorías seleccionadas.

Los motivos tenían que ver con las razones que movieron a los estudiantes a elegir la modalidad, la escuela y la especialidad. La valoración de la formación recibida era una evaluación que el grupo de estudiantes realizaba de su paso por la institución. Las expectativas futuras, como señala Córica (2015), permitieron relevar las condiciones que visualizaban como posibles y las oportunidades que proyectaban como realizables. 


\section{Contexto}

El sector productivo más dinámico de la provincia es el forestal. En la provincia de Corrientes existen 474,000 hectáreas plantadas con especies de rápido crecimiento (pinos y eucaliptos) (Elizondo et al., 2015), lo que la convierte en la provincia mesopotámica con mayor superficie. Actualmente, posee el 38\% y, junto con Misiones y Entre Ríos, suman el 80\% de la superficie forestada de la Argentina (Corrientes, Gobierno Provincial, 2013).

Esto genera 35,000 puestos de trabajo directos e indirectos (Solari et al., 2012). Sin embargo, Corrientes se encuentra en una etapa incipiente dentro de las distintas fases de desarrollo posible de un complejo foresto-industrial (Peirano, Bustos y Nahirñak, 2009). La situación laboral de los trabajadores, en general, se caracteriza por ser precaria y de baja intensidad. En el sector aserradero solo el $40 \%$ están empleados en establecimientos con un proceso industrial integrado. El 47\% no tiene seguro a través de la Aseguradora de Riesgos del Trabajo (ART). En las carpinterías, el grado de informalidad en la que operan es alto, como lo es también el grado de baja calificación. Por otra parte, el sector foresto-industrial se caracteriza por la presencia de puestos laborales de alto riesgo (Peirano, 2015).

Con el objetivo de planificar el futuro de la provincia, dentro del Plan Estratégico Participativo (PEP2021), en 2012 se inició un plan estratégico para el sector forestoindustrial de la provincia de Corrientes (PEFIC). Uno de los nudos críticos identificados fue la baja disponibilidad de recursos humanos calificados en el sector, tanto a nivel operativo como gerencial. Entonces, entre otras acciones, se propuso la especialidad de Industrialización de la Madera y el Mueble en seis EST del interior de la provincia.

La EST elegida nació como Escuela de Aprendizaje y Medio Turno en 1948. El primer curso fue inaugurado con 70 alumnos varones, con la especialidad de Ajustadores y Torneros, la que se complementaba con la enseñanza de Historia, Geografía, Aritmética y Geometría. A partir de 1950 pasó a denominarse Escuela Fábrica durante diez años; luego, desde 1961, Escuela Nacional de Educación Técnica. En 1982 ingresaron por primera vez las mujeres.

En este momento ofrece ciclo básico y ciclo superior de educación secundaria. Además, tiene anexo un centro de formación profesional. En el ciclo superior ofrece tres especialidades: Electromecánica, Construcciones e Industrialización de la Madera y el Mueble. La última empezó a ofrecerse a partir de 2013 en consonancia con el desarrollo previsto por el PEFIC. Esto quiere decir que, en 2017, se recibió la segunda promoción. En ese año, la escuela tenía una matrícula de 993 estudiantes en la secundaria ( $82 \%$ de varones y $18 \%$ de mujeres), de los cuales 50 cursaban la especialidad Madera y Muebles (74\% de varones y $26 \%$ de mujeres). Como señalamos, para ese entonces, seis EST ofrecían la especialidad en la provincia, de las cuales la escuela elegida era la que más matrícula reportaba.

\section{Población}

De los 50 estudiantes que cursaban la especialidad, 42 respondieron la encuesta (84\%), 36 varones (86\%) y 6 mujeres (14\%). La mayoría tenía entre 15 y 24 años 
(88\%). El clima educativo del hogar de casi la mitad de los encuestados (40\%) es bajo. Esto implica que el máximo nivel educativo alcanzado en el hogar por alguno de los adultos (madre, padre, abuelos, otros) con quienes convive no llega a secundaria completa. Esto significa que, seguramente, estos estudiantes serán la primera generación que recibirá una certificación del nivel.

El 43\% de los encuestados no respondieron sobre la ocupación del padre. Entre los que respondieron, hay una variedad de ocupaciones y destacan los que se dedican a la construcción (14\%). Solo uno está vinculado al sector de la madera. El $38 \%$ no respondió acerca de la ocupación de la madre. De los que sí lo hicieron, un $31 \%$ mencionó que la madre era ama de casa. Después aparecen las ocupaciones tradicionalmente femeninas: empleada doméstica, costurera, peluquera, manicura, maquilladora, secretaria.

\section{RESULTADOS Y DISCUSIóN}

\section{El ingreso a la escuela técnica y a la especialidad}

La principal motivación de elección de la modalidad de ETP es la expectativa de rápida inserción laboral y, luego, la posibilidad de continuar estudios superiores. En este orden de prioridades, no se diferencian varones y mujeres. En los primeros, estas elecciones se manifiestan en un 64 y 14\%, respectivamente, y en las segundas, en un 50 y $33 \%$ (ver tabla 1). Esto se condice con resultados de otras investigaciones, que indican que, en la ETP, los jóvenes no siempre deciden estudiar por una cuestión vocacional, sino que responden más bien a factores económicos. Además, confirman que la decisión no es autónoma; es el sistema el que empuja a tomar esa determinación (Sweet, 2006). En este caso, el gobierno (provincial y nacional) ha dado un importante impulso al desarrollo del sector.

Tabla 1. Principal motivo por el que se eligió la modalidad técnico-profesional por sexo

(Corrientes, Argentina, 2017)

\begin{tabular}{|c|c|c|c|c|}
\hline \multirow{2}{*}{ Motivos } & & \multicolumn{2}{|c|}{ Sexo } & \multirow{2}{*}{ Total } \\
\hline & & Varón & Mujer & \\
\hline \multirow{2}{*}{$\begin{array}{l}\text { 1. Porque la formación técnica brinda mayores posibilidades } \\
\text { de conseguir un trabajo }\end{array}$} & $\mathrm{N}$ & 23 & 3 & 26 \\
\hline & $\%$ & 63.9 & 50.0 & 61.9 \\
\hline \multirow{2}{*}{$\begin{array}{l}\text { 2. Porque quiero seguir una carrera terciaria/universitaria } \\
\text { relacionada con la formación técnica }\end{array}$} & $\mathrm{N}$ & 5 & 2 & 7 \\
\hline & $\%$ & 13.9 & 33.3 & 16.7 \\
\hline \multirow{2}{*}{ 3. Porque me gusta la formación práctica } & $\mathrm{N}$ & 4 & 1 & 5 \\
\hline & $\%$ & 11.1 & 16.7 & 11.9 \\
\hline \multirow{2}{*}{ 4. Por tradición familiar } & $\mathrm{N}$ & 2 & 0 & 2 \\
\hline & $\%$ & 5.6 & 0 & 4.8 \\
\hline \multirow{2}{*}{ 5. Porque no había otra opción } & $\mathrm{N}$ & 2 & 0 & 2 \\
\hline & $\%$ & 5.6 & 0 & 4.8 \\
\hline \multirow{2}{*}{ Total } & $\mathrm{N}$ & 36 & 6 & 42 \\
\hline & $\%$ & 100.0 & 100.0 & 100.0 \\
\hline
\end{tabular}

Fuente: Elaboración propia a partir de encuesta. 
Algunas mujeres manifiestan la falta de apoyo familiar frente a la carrera elegida, ya que existe la percepción de que la educación técnica está relacionada con la fuerza física, lo que nos lleva a concebir que estos estudios se orientan hacia determinados tipos de trabajo o especialidades técnicas que, históricamente, han sido pensados como espacios de formación para hombres.

La importancia del contexto familiar en estas elecciones "atípicas" de especialidad por parte de mujeres también fue revelada en una investigación de Pérez (2012) sobre escuelas técnicas de la ciudad de Córdoba. La amplia mayoría de mujeres entrevistadas tenían algún familiar que había concurrido al mismo establecimiento o se dedicaba a una profesión de orientación técnica. Sin embargo, las mujeres no siempre fueron apoyadas por sus familias en la elección. Exactamente lo opuesto les ocurría a los estudiantes varones. En este punto, la variable generacional es muy significativa. Bloj (2017) evidencia que, en las mujeres entre 35 y 50 años de edad, la preferencia por la ETP ha sido más resistida en el ámbito social y el entorno familiar que en el caso de las mujeres de entre 20 y 35 años. Ello obedece no solo a que estas últimas han transitado su formación en un periodo de revalorización de la ETP, sino a un contexto social con mayores derechos consagrados en materia de equidad de género.

Una investigación de Milleneaar (2017) sobre políticas de empleo con enfoque de género da cuenta de la falta de apoyo familiar cuando las mujeres intentan incursionar en cursos considerados masculinos. En este sentido, sostiene la autora que si ya es complejo convocar a mujeres a este tipo de formación, las resistencias encontradas en sus vínculos personales y familiares vuelven aún más difícil la decisión de sostener el curso. Sin embargo, en nuestro caso advertimos cómo las mujeres pudieron resistir a los mandatos familiares y lograron su objetivo: "Mi papá, por ejemplo, no quería. Decía que esta escuela era de hombres. Y yo le decía que quería venir" (estudiante mujer).

En cuanto a los principales motivos por los que eligieron la escuela en la que estudian, la mayoría de los varones respondieron que es por la orientación/especialización (69\%); en cambio, las opiniones de las mujeres están divididas entre las que manifestaron lo mismo (50\%) y las que eligieron esta institución porque es una buena escuela (50\%) (ver tabla 2).

Tabla 2. Principal motivo por el que se eligió esta escuela por sexo (Corrientes, Argentina, 2017)

\begin{tabular}{|c|c|c|c|c|}
\hline \multirow{2}{*}{ Motivos } & & \multicolumn{2}{|c|}{ Sexo } & \multirow{2}{*}{ Tota } \\
\hline & & Varón & Mujer & \\
\hline \multirow{2}{*}{ 1. Por la orientación/especialización que ofrece } & $\mathrm{N}$ & 25 & 3 & 28 \\
\hline & $\%$ & 69.4 & 50.0 & 66.7 \\
\hline \multirow{2}{*}{ 2. Porque es una buena escuela } & $\mathrm{N}$ & 3 & 3 & 6 \\
\hline & $\%$ & 8.3 & 50.0 & 14.3 \\
\hline \multirow{2}{*}{ 3. Porque me la recomendaron } & $\mathrm{N}$ & 6 & 0 & 6 \\
\hline & $\%$ & 16.7 & .0 & 14.3 \\
\hline \multirow{2}{*}{ 4. Porque queda cerca de mi casa } & $\mathrm{N}$ & 2 & 0 & 2 \\
\hline & $\%$ & 5.6 & .0 & 4.8 \\
\hline \multirow{2}{*}{ Total } & $\mathrm{N}$ & 36 & 6 & 42 \\
\hline & $\%$ & 100.0 & 100.0 & 100.0 \\
\hline
\end{tabular}

Fuente: Elaboración propia a partir de encuesta. 
Respecto a los motivos de elección de la especialidad Industrialización de la Madera y el Mueble, un tercio de los varones contestaron que fue porque les ayudaría a conseguir trabajo y otro tercio, porque les gusta. En cambio, la mitad de las mujeres aseguraron que lo hicieron porque creían que les ayudaría a conseguir trabajo (50\%) y ninguna dijo que lo hizo porque le gustaba (ver tabla 3 ).

Tabla 3. Principal motivo por el que se eligió la especialidad por sexo (Corrientes, Argentina, 2017)

\begin{tabular}{|c|c|c|c|c|}
\hline \multirow{2}{*}{ Motivos } & & \multicolumn{2}{|c|}{ Sexo } & \multirow{2}{*}{ Total } \\
\hline & & Varón & Mujer & \\
\hline \multirow{2}{*}{ 1. Porque creo que me va a ayudar a conseguir trabajo } & $\mathrm{N}$ & 13 & 3 & 16 \\
\hline & $\%$ & 36.1 & 50.0 & 38.1 \\
\hline \multirow{2}{*}{ 2. Porque me gusta } & $\mathrm{N}$ & 13 & 0 & 13 \\
\hline & $\%$ & 36.1 & .0 & 31.0 \\
\hline \multirow{2}{*}{ 3. Porque me ayuda a capacitarme en mi trabajo actual } & $\mathrm{N}$ & 3 & 1 & 4 \\
\hline & $\%$ & 8.3 & 16.7 & 9.5 \\
\hline \multirow{2}{*}{$\begin{array}{l}\text { 4. Porque me prepara para la carrera terciaria/universitaria que } \\
\text { quiero seguir }\end{array}$} & $\mathrm{N}$ & 4 & 2 & 6 \\
\hline & $\%$ & 11.1 & 33.3 & 14.3 \\
\hline \multirow{2}{*}{ 5. Porque para los varones es una buena formación práctica } & $\mathrm{N}$ & 2 & 0 & 2 \\
\hline & $\%$ & 5.6 & .0 & 4.8 \\
\hline \multirow{2}{*}{ 6. Otros motivos } & $\mathrm{N}$ & 1 & 0 & 1 \\
\hline & $\%$ & 2.8 & .0 & 2.4 \\
\hline \multirow[t]{2}{*}{ Total } & $\mathrm{N}$ & 36 & 6 & 42 \\
\hline & $\%$ & 100.0 & 100.0 & 100.0 \\
\hline
\end{tabular}

Fuente: Elaboración propia a partir de encuesta.

Como veremos a continuación, la información presentada también la encontramos en los jóvenes que participaron en los grupos focales:

Vengo de una familia de técnicos y eso de un principio me dio curiosidad por saber lo que saben mi tío y mi hermano que se recibieron acá [...] Después escuché una charla de la tecnicatura y la verdad que me re gustó... (estudiante varón).

Cuando era chico veía Discovery y vería que hacían casas de madera y ése fue el primer incentivo... (estudiante varón).

Porque me parece útil [...] al ser que estamos en Corrientes que hay mucho para explotar así que vamos a tener inserción laboral (estudiante mujer).

Tanto varones como mujeres reconocen que, en el momento del ingreso, la escuela no hizo diferencias de género respecto a la especialidad y las oportunidades futuras; en cambio, sí lo hizo en la convivencia escolar (como la vestimenta y otras costumbres). Esto es recordado principalmente por los varones (69\%). En consecuencia, la gestión del ingreso es un aspecto fundamental a partir del cual se promueve, o no, la democratización en el acceso a la ETP. Sin embargo, las estrategias que favorecen tal democratización no son tan claras.

\section{Valoración de la formación recibida}

Varones y mujeres valoran de manera positiva la formación recibida en la escuela técnica, sobre todo el trabajo que realizan en el taller. En este espacio adquieren competencias que les ayudan a desempeñarse en sus propios hogares e iniciarse en el 
ámbito laboral: "En la parte práctica es la mejor [...]. La parte práctica me gusta más" (estudiante varón). "Investigadora: ¿Qué cosas hacen en sus casas que aprenden acá? Estudiante: Instalaciones, la parte de electricidad usamos mucho” (estudiante mujer).

Tanto a varones (86\%) como a mujeres (67\%) les gustaría que la escuela los preparara en habilidades técnicas como aplicar tecnologías a tareas específicas, utilizar herramientas para el desempeño de un trabajo, etcétera. Sin embargo, el 100\% de las mujeres consideraron que la escuela técnica las prepara principalmente en habilidades sociolaborales, como resolver problemas prácticos y trabajar en equipo, mientras que un 50\% pensaron que lo hace en capacidades analíticas (pensar de modo creativo, tomar decisiones, solucionar problemas, usar imaginación, saber razonar). En cambio, para la mayoría de los varones (83\%), la escuela cumple con sus expectativas (ver tabla 4).

Tabla 4. Expectativas y valoraciones de la formación por sexo (Corrientes, Argentina, 2017)

\begin{tabular}{|c|c|c|c|c|}
\hline & \multicolumn{2}{|c|}{ Expectativa } & \multicolumn{2}{|c|}{ Realidad } \\
\hline & Varones & Mujeres & Varones & Mujeres \\
\hline $\begin{array}{l}\text { En habilidades técnicas (aplicar tecnologías a tareas específi- } \\
\text { cas, utilizar herramientas para el desempeño de un trabajo) }\end{array}$ & 86.1 & 66.7 & 83.3 & 50 \\
\hline $\begin{array}{c}\text { En habilidades sociolaborales (resolver problemas prácticos, } \\
\text { trabajar en equipo) }\end{array}$ & 47.2 & 50 & 55.6 & 100 \\
\hline $\begin{array}{c}\text { En capacidades analíticas (pensar de modo creativo, tomar } \\
\text { decisiones, solucionar problemas, usar la imaginación, saber } \\
\text { razonar) }\end{array}$ & 63.9 & 33.3 & 38.9 & 50 \\
\hline $\begin{array}{l}\text { En capacidades básicas (lectura, redacción, aritmética y mate- } \\
\text { mática, expresión oral) }\end{array}$ & 30.6 & 50 & 61.1 & 0 \\
\hline $\begin{array}{l}\text { En cualidades personales (responsabilidad, autoestima, socia- } \\
\text { bilidad, autocontrol) }\end{array}$ & 33.3 & 66.7 & 27.8 & 16.7 \\
\hline
\end{tabular}

Fuente: Elaboración propia a partir de encuesta.

En cuanto a la pregunta de cómo debería formar la escuela técnica a varones y mujeres, el 100\% de las mujeres y el $97 \%$ de los varones consideraron que a todos por igual. El 68\% de las mujeres se manifestaron de manera neutral respecto a la consulta sobre si la educación técnica es más adecuada para los varones. En cambio, en el caso de los varones, el $42 \%$ dijeron que sí lo es.

Cuando la pregunta es la contraria, si la educación técnica es más adecuada para las mujeres, la mitad de las mujeres (50\%) poseen una visión neutral al respecto y el $52.8 \%$ de los varones opinaron que no. Analizando lo que predomina en la mayoría de las respuestas, advertimos una percepción estereotipada del género. En la misma localidad y a pocas cuadras, hay otra escuela técnica donde la mayoría son mujeres, pero esto parece que no lo tienen en cuenta.

En las encuestas, la mayoría de los varones (86\%) y la mitad de las mujeres (50\%) señalaron que en los talleres ambos son tratados de la misma manera. Sin embargo, cuando se refirieron a los docentes, estos resultados dieron vuelta, ya que para la mayoría de las mujeres (83\%) no hay diferencias en cuanto a las exigencias y solo la mitad de los varones sostuvieron esto (50\%). Ambos grupos coincidieron en que las maquinarias y la tecnología son utilizadas del mismo modo (75\% de varones y $83 \%$ de mujeres).

En los grupos focales, tanto varones como mujeres se autopercibieron en igualdad de condiciones. Sin embargo, a diferencia de la encuesta, comentaron que los 
docentes de los talleres (no los de teoría) generan distinciones en función del tipo de tareas a desarrollar, vinculadas de preferencia a la fuerza. Este trato diferencial es entendido como una cuestión de "caballerosidad" y "cuidado" por parte de los varones, mientras que para las mujeres es discriminatorio al no ser formadas en la práctica como sus pares masculinos. Para Pérez (2012), el género es construido en medio de contextos sociales y culturales que producen múltiples formas de masculinidad, una de las cuales ejerce hegemonía sobre las otras. Así, los hombres se ven como los fuertes, protectores y aptos para todo tipo de trabajos:

Investigadora: ¿Qué implica ser hombre acá?

Estudiante: Prácticamente implica lo de siempre, mucha fuerza (estudiante varón).

Investigadora: ¿Pero ustedes por su propia forma de ser hacen lo más pesado? Estudiante: Sí, por caballerismo.

Investigadora: ¿Por eso? ¿O porque piensan que las chicas no podrán?

Estudiante: No, por caballeros. Porque en los cortes, en los usos de máquinas les hacen hacer lo mismo (estudiante varón).

Nuestros compañeros [haciendo referencia a los varones] no hacen diferencias [...] te acompañan, te ayudan en todo. Creo que los chicos son más atentos. En eso por ahí se nota más [las diferencias] en algunas actitudes que tiene un profesor en el taller... (estudiante mujer).

Los docentes hacen diferencias [...]. A mí no me gusta. Quiero hacer lo mismo [...]. Por ejemplo, los varones hacen la mezcla y a nosotras nos dan una planilla para registrar [...]. Yo puedo ser mujer y hacer lo mismo que vos (estudiante mujer).

Estos relatos refuerzan la idea de que el sexismo presente en las aulas y en las interacciones docentes-estudiantes es una parte del contenido que la escuela promueve. Según León (2009), en la EST abundan los registros de subestimación y desvalorización del personal docente y directivo sobre las alumnas, manifestados en las prácticas educativas. En muchos casos, se les prohíbe manipular máquinas o se les asignan tareas diferentes por el mero hecho de ser mujeres. Se muestra cómo el tratamiento a las mujeres por parte de docentes y compañeros varía entre el tutelaje (se debe cuidarlas porque son diferentes) a la desvalorización de sus conocimientos y habilidades. No solo en la transmisión se reproduce un modelo sexista, sino también cada vez que se recortan las expectativas sobre las mujeres o cuando se magnifican, en función de una competencia entre los sexos, en la que las mujeres están obligadas a demostrar que su rendimiento es análogo al de los varones (Seoane, 2013): "Yo siento que es como para no te lastimes, porque tenemos que estar llevando vigas de un galpón a otro y te dicen: 'No, llevá una que te vas a forzar'. Y yo de caprichosa llevo tres" (estudiante mujer).

Las prácticas que generan inequidades pueden ser legitimadas a través de argumentos relativos a roles de género o hasta diferencias biológicas entre hombres y mujeres. Estas diferencias pueden asociarse al llamado "sexismo benevolente" que estereotipa a las mujeres describiéndolas como maravillosas, pero débiles, por lo que necesitan protección masculina (Glick \& Fiske, 1996).

A lo largo de la historia se ha impuesto un ideal masculino vinculado a la potencia y superioridad, en contraste con la debilidad y sumisión asociada a la posición 
femenina. Este ideal orienta e interpela a los individuos concretos y los constituye como sujetos. Sin embargo, es necesario resaltar la heterogeneidad de experiencias biográficas y subjetivas de quienes llegan a esas aulas (Millenaar, 2019).

Casi la mitad de los varones (46\%) y la mitad de las mujeres (50\%) no consideran que los varones sean más inteligentes y las mujeres más aplicadas. En cambio, la mitad de ambos grupos (50\%) piensa que los varones tienen más habilidades manuales, pero las mujeres escriben mejor.

Bloj (2017) sostiene que las estudiantes de ETP sufren, durante la formación, que se les adjudiquen las supuestas "cualidades naturales" de las mujeres, como la prolijidad y el detalle; también por la necesidad de demostrar que pueden participar en los talleres en paridad con los hombres. A la par del discurso "homogeneizador", centrado en el trato igualitario de hombres y mujeres y que gira en torno a la idea de que, dentro de la escuela técnica, "las chicas lijan, levantan paredes, revocan, igual que los hombres", en su estudio se resaltan situaciones que contradicen estas afirmaciones.

Tal como expresamos en los apartados anteriores, se evidencia un intento para que las discriminaciones pasen "inadvertidas" como tales, cuando no son negadas de manera directa, y estas se evidencian en diferentes representaciones y prácticas. Cabe destacar, inicialmente, los llamados "códigos masculinos" que circulan en la ETP y que condensan significaciones sedimentadas. Si bien en los procesos de enseñanza-aprendizaje se producen interacciones, conflictos y alianzas, predomina un estilo de relación entre varones y mujeres que impone a estas últimas "adaptarse a esos códigos" (Bloj, 2017). Estas barreras no siempre son reconocidas por las propias mujeres y muchas se encuentran plegadas al imaginario dominante, condicionadas por los estereotipos, que minimizan las desigualdades.

Así, los códigos de género se manifiestan, ante todo, en los talleres a través del lenguaje, las prácticas de enseñanza y el modo en que se relaciona el estudiantado.

Sumado a esto, estudiantes de ambos sexos valoran la socialización con sus pares. Dubet (2006) señala que las experiencias más importantes para la juventud ocurren en los intersticios de las instituciones, donde tienen lugar relaciones de sociabilidad, de ahí que las escuelas de nivel secundario (técnicas y escuelas secundarias orientadas) se convierten en los lugares privilegiados de la vida juvenil. Así lo expresan los estudiantes: "[Valoro] la experiencia de compartir con compañeros [refiriéndose a ambos sexos]" (estudiante varón). "Fui muy feliz con mis compañeros [tanto varones como mujeres], casi conviví con ellos" (estudiante mujer).

\section{Expectativas laborales y educativas}

Tanto los varones como las mujeres de nuestro estudio, en lo referente a los escenarios laborales futuros, aducen que las mujeres no tendrán las mismas oportunidades que los varones por más que tengan la misma formación debido a la mentalidad de los empleadores. Al respecto, como señalamos en páginas anteriores, Bloj (2017) asegura que los estereotipos culturales y las prácticas hegemónicas que atraviesan a hombres y a mujeres, y que circulan en estos ámbitos, son una de las principales barreras para el acceso al mercado de trabajo y, en los casos en que se sortean, obligan a las mujeres a "demostrar" capacidades de modo constante: 
No sé por qué se ve que la mujer tiene que estar de recepcionista en una oficina o algo de eso y el hombre es el que tiene que estar trabajando de electricista y esas cosas [...], yo creo que es por eso, eso es lo que tiene la gente en la cabeza, eso es lo que cree (estudiante mujer).

El 67\% de las mujeres creen que podrían desempeñarse al finalizar la escuela en otro tipo de trabajo en el que apliquen algunos conocimientos aprendidos en la especialidad, mientras que el $47.2 \%$ de los varones consideran que trabajarían en algo vinculado a la especialidad. Esto tiene que ver con las posibilidades de inserción laboral antes manifestadas.

Los estudios de inserción de egresados muestran que, en general, los graduados de EST tienen mejores condiciones y más rápido ingreso al mercado de trabajo (Sosa, 2016), así como diferencias según el sexo entre los egresados/as de EST; concluyen que los varones presentan ventajas respecto a las mujeres, no solo sobre la tasa de desocupación, sino también la calidad del empleo y los niveles de calificación de los puestos a los que acceden (Sosa, 2018). Nosotros consultamos acerca de las expectativas y los estudios de trayectorias laborales podrán confirmar o refutar esto.

Respecto a las expectativas futuras de formación, mientras los varones manifiestan interés por seguir estudiando, las mujeres no mencionan este tema: "A mí me gustaría. Sería un plus de lo que yo hago. Es como que lo que sé ya agoté y querría hacer otras cosas" (estudiante varón). "A mí me gustaría ser marinero y después seguir estudiando" (estudiante varón).

En cuanto a sus expectativas a futuro, las investigaciones muestran diferencias en torno al género. En primer lugar, una diferenciación proviene del valor del título técnico para la inmediata inserción laboral. La necesidad de pasar por la escuela secundaria para conseguir trabajo y la urgencia de entrar en el mercado laboral de manera rápida se reconocen como rasgo característico de los varones, y no se observa de forma tan fuerte entre las mujeres, que, en ocasiones, priorizan la continuidad de los estudios universitarios, una vez finalizada la escuela secundaria. De acuerdo con Pérez (2012), ello se explica desde el mandato de "proveedor" y "sostén", que obedece a la idea de masculinidad, generada y difundida en la sociedad y en los medios de comunicación.

A diferencia de lo anterior, los estudiantes de la EST analizada pertenecen a una localidad del interior de la provincia, donde no hay ninguna universidad. Cuentan con algunas extensiones universitarias y ofertas de educación terciaria, que no siempre se condicen con sus intereses. Además, poseen poca información respecto a la continuación de estudios superiores en otras localidades.

\section{CONCLUSIONES}

En los últimos años ha existido un renovado interés en la ETP por su importancia para el desarrollo productivo. La posibilidad que creó la Ley Nacional de Educación Técnico Profesional 26.058 de 2005, de contar con fondos, procesos de fortalecimiento institucional y homologación de títulos y certificaciones, cambió las condiciones de funcionamiento de las EST y los centros de formación profesional. A las modificaciones normativas se sumó la mayor demanda social en torno a los derechos de las mujeres, lo que constituye un trasfondo en el cual las instituciones de esa modalidad intervienen con sus propuestas educativas. No obstante, la ETP continúa 
reflejando procesos de reproducción de las desigualdades de género y escasa problematización de esta, aun cuando se reconoce el crecimiento de la matrícula femenina. Las problemáticas reconocidas y trabajadas a nivel institucional se vinculan a otros temas, por ejemplo, la permanencia de estudiantes.

Con este artículo nos propusimos comprender las reflexiones de los jóvenes que cursan la especialidad Madera y Muebles en una escuela técnica del interior de la provincia de Corrientes (Argentina) acerca de la educación y el trabajo, y cómo estos temas están atravesados por la categoría género. Las encuestas y los grupos focales nos permitieron indagar las experiencias de los estudiantes en las dinámicas de escolarización y género, y relevaron los motivos de la elección de la escuela técnica y la especialidad, la valoración de la formación recibida y sus expectativas futuras en relación con el estudio y el trabajo.

Históricamente, la escuela técnica se encargó de formar trabajadores a través de códigos de género vinculados al ideal de superioridad, fuerza física, provisión y protección. La naturalización de ciertas características asociadas al género (los hombres utilizan "la fuerza y desafían el peligro", las mujeres son "más prolijas y ordenadas") parecen tener incidencia en las acciones formativas y las actividades prácticas en los talleres de especialidad. El trato privilegiado y la protección brindada a las estudiantes mujeres emergían en algún grado de un "sexismo benevolente", que es una forma de discriminación, pero al mismo tiempo socavaban el estatus de las mujeres en determinadas situaciones y posiciones.

Si bien el acceso de mujeres a esta especialidad no está vedado, la cultura masculina observada en la EST es análoga a la del mercado laboral, que, finalmente, plantea barreras a la participación femenina. Esto se refuerza con la socialización familiar y del contexto social próximo que antecede y acompaña las trayectorias escolares. Algunas estudiantes manifiestan la falta de apoyo familiar, sobre todo de parte del progenitor masculino, frente a la elección de la carrera elegida y también de la institución.

Sin embargo, es necesario resaltar la heterogeneidad de experiencias biográficas y subjetivas de quienes llegan a la EST. En este sentido, el análisis de la desigualdad estructural de género y el registro de los códigos presentes en las instituciones deben complementarse con el modo en que varones y mujeres se apropian, cuestionan o resisten esos códigos, al encarnarlos de maneras diversas en sus propios recorridos. Así, observamos jóvenes mujeres con una visión no estereotipada del género que resisten los mandatos sociales.

Más allá del lugar logrado por los temas de género en la agenda pública, con repercusiones en las políticas y acciones en desarrollo, su implementación a nivel de las instituciones continúa planteando el desafío de que las propias escuelas técnicas y sus actores consideren la cuestión de género como una temática y problemática que está presente, aunque a veces invisibilizada u oculta.

Proponemos el diseño de estrategias que favorezcan la democratización a fin de lograr una mayor participación e inclusión de mujeres tanto en el ingreso como en la selección de la especialidad. El protagonismo docente a partir de su capacitación en los temas relacionados con el género puede ser la base desde la cual se inicien cambios en el sistema social y cultural. 
Entendemos que las políticas de formación no tienen la responsabilidad de la generación de empleo, pero sí podrían apoyar a las personas para que se transformen en identificadoras de oportunidades, y pasen del estatus de sujetos pasivos, dependientes de una intervención externa que los acerque a una oferta de trabajo insuficiente, al de constructoras de su estrategia de empleabilidad. También, creemos que es responsabilidad de las instituciones educativas propiciar el diálogo y diseñar estrategias de vinculación con los sectores productivos (espacios de prácticas educativas, capacitación, etcétera) a fin de habilitar la inclusión y participación de las mujeres. Sería interesante poder trabajar con los empleadores el mismo trabajo acerca de los estereotipos y prejuicios que proponemos para los docentes. En ambos casos implica un cambio considerable en valores, creencias y roles sobre el trabajo, y representa un desafío para la sociedad en conjunto, en el sentido de que habría que reforzar los vínculos entre los ministerios de Educación, el Ministerio de Industria, Trabajo y Comercio, y el Ministerio de Producción para establecer una agenda de trabajo que aborde la equidad de género.

Con este trabajo esperamos contribuir con conocimientos sobre un tema poco estudiado en la academia: las mujeres en trayectos formativos y laborales tradicionalmente masculinos. Además, apostamos, en sintonía con los Objetivos de Desarrollo Sostenible de la $\mathrm{ONU}$, a empoderar y recuperar las palabras de las personas jóvenes como protagonistas de estos espacios.

\section{REFERENCIAS BIBLIOGRÁFICAS}

Argentina. Instituto Nacional de Educación Tecnológica (INET) (2018). Mujeres en la ETP: desigualdades y oportunidades. Buenos Aires: INET.

Argentina. Instituto Nacional de Estadísticas y Censos (INDEC) (2010). Censo Nacional de Población y Vivienda. Buenos Aires.

Argentina. Poder Legislativo (2005). Ley de Educación Técnico-Profesional 26.058. Buenos Aires.

Bloj, Cristina (2017). Trayectorias de mujeres. Educación técnico-profesional y trabajo en la Argentina. Cepal, serie Asuntos de Género núm. 145. Santiago: Naciones Unidas.

Bourdieu, Pierre (1998). Capital cultural, escuela y espacio social (2ạ ed.). Madrid: Siglo XXI.

Conde, Analaura (2013). Educación técnica y género en Uruguay: ¿una realidad que se transforma? Tesis de maestría, Universidad de la República de Uruguay.

Córica, Agustina (2015). Juventud y futuro: expectativas educativas y laborales de los estudiantes de la escuela secundaria. Presentado en el 12음 Congreso Nacional de Estudios del Trabajo. El Trabajo en su Laberinto. Viejos y Nuevos Desafíos. Buenos Aires, Argentina. Universidad de Buenos Aires, Facultad de Ciencias Económicas, Asociación Argentina de Especialistas en Estudios del Trabajo (ASET). Recuperado de http://www.aset.org.ar/2015/ponencias/7_ Corica.pdf

Corrientes. Gobierno Provincial (2013). Plan Estratégico Foresto Industrial de la Provincia de Corrientes. Corrientes. Recuperado de http://itc.corrientes. gob.ar/noticia/plan-estrategico-foresto-industrial-de-corrientes. 
D’Andrea, Ana María (2018). La formación para el trabajo en el sector foresto-industrial para jóvenes de la provincia de Corrientes (Argentina). En P.A. Vommaro, A. Barcala y L. Rangel (comps.). Derechos y políticas en infancias y juventudes: diversidades, prácticas y perspectivas (pp. 165-208). Buenos Aires: Consejo Latinoamericano de Ciencias Sociales. Manizales: Universidad de Manizales. Sabaneta: Centro Internacional de Educación y Desarrollo Humano.

D’Andrea, Ana María y Buontempo, María Paula (2018). Las tramas de las políticas públicas de formación para el trabajo en el sector foresto-industrial en la localidad de Virasoro (Corrientes, Argentina). RELAPAE. Revista Latinoamericana de Políticas y Administración de la Educación, vol. 5, núm. 8, pp. 4762. Recuperado de http://relapae.com.ar/wp-content/uploads/relapae_5_8_ dandrea_buontempo_formacio\%CC\%81n_trabajo_argentina.pdf

Dubet, François (2006). El declive de la institución. Profesiones, sujetos e individuos ante la reforma del Estado. Barcelona: Gedisa.

Dubet, François y Martuccelli, Danilo (1998) En la escuela. Sociología de la experiencia escolar. Buenos Aires: Losada.

Elizondo, M. H. et al. (2015). Actualización del inventario de plantaciones forestales de la provincia de Corrientes. Corrientes: Gobierno de la Provincia, Consejo Federal de Inversiones. Recuperado de http://recursosforestales.corrientes.gob.ar/assets/articulo_adjuntos/1777/original/Informe_Final_Inventario_2015.pdf?1450792766

Evans, Karen (2006). Achieving equity through 'gender autonomy': The challenges for VET policy and practice. Journal of Vocational Education \& Training, vol. 58.

Gallart, María Antonia, Miranda, Martín, Peirano, Claudia y Sevilla, María Paola (2003). Tendencias de la educación técnica en América Latina. Estudios de casos en Argentina y Chile. Unesco, Instituto Internacional de Planeamiento Educativo.

Gamba, Susana (cood.) (2007). Diccionario de estudios de género y feminismos. Buenos Aires: Biblos.

Glick, Peter \& Fiske, Susana (1996). The ambivalent sexism inventory: Differentiating hostile and benevolent sexism. Journal of Personality and Social Psychology, vol. 70, núm. 3, pp. 491-512. Recuperado de http://citeseerx.ist. psu.edu/viewdoc/download?doi=10.1.1.470.9865\&rep=rep1\&type=pdf

Jackson, Philips. (2001). La vida en las aulas. Madrid: Morata.

Lamas, Marta (comp.) (2000). El género. La construcción cultural de la diferencia sexual. México. UNAM: Miguel Ángel Porrúa.

Lavigne, Luciana (2009). Entre el deseo y la regulación: poéticas y políticas del rostro. Ponencia presentada en VIII RAM. Buenos Aires, ICA UBA.

León, Fernanda Graciela (2009). Mujeres y discurso pedagógico en la escuela técnica. En Alejandro Villa (comp.). Sexualidad, relaciones de género y de generación. Perspectivas histórico-culturales en educación (pp. 171-199). Buenos Aires: Noveduc.

Millenaar, Verónica (2019). Masculinidades en la formación profesional: expectativas y sentidos del trabajo en un contexto de incertidumbre laboral. Descentrada, vol. 3, núm. 1. https://doi.org/10.24215/25457284e069

Millenaar, Verónica (2018). Mujeres y varones ¿caminos laborales diferentes? La orientación y la formación para el trabajo en la escuela secundaria desde 
una perspectiva de género. En Silvia Martínez (comp.). Conversaciones en la escuela secundaria. Política, trabajo y subjetividad (pp. 141-160). Neuquén: Universidad Nacional del Comahue.

Milleneaar, Verónica (2017). Políticas de empleo con enfoque de género: formación laboral en oficios no tradicionales para mujeres. Cadernos Pagu, vol. 51. doi: 10.1590/18094449201700510014

Millenaar, Verónica (2016). Trayectorias educativo-laborales de varones y mujeres jóvenes de sectores populares que participan de dispositivos de formación para el trabajo (Área Metropolitana de Buenos Aires, 2008-2014). Entre la profesionalización, la acumulación y la socialización. Tesis de doctorado en Ciencias Sociales, Universidad de Buenos Aires.

Morgade, Graciela (2001). Aprender a ser mujer. Aprender a ser varón. Buenos Aires: Novedades Educativas.

Organización de las Naciones Unidas (ONU) (2015) Objetivos de Desarrollo Sostenible. Recuperado de http://www.un.org/sustainabledevelopment/es/ objetivos-de-desarrollo-sostenible/

Peirano, Claudia (2015). Sin excusas. Avances en la formación continua y la certificación de los trabajadores en el sector forestal y de la madera. Foresto Industria. La Revista Foresto Industrial del Ministerio de Agricultura, Ganadería y Pesca, vol. 5, núm. 12, pp. 11-14. Recuperado de http://forestoindustria. magyp.gob.ar/_archivos/_revistas/revista12.pd

Peirano, Claudia, Bustos Hinostroza, Misael y Nahirñak, Paula (2009). Recursos humanos en el sector forestal. Un análisis cuantitativo y cualitativo a partir del Programa de Certificación de Competencias Laborales. Presentado en el XIII Congreso Forestal Mundial. Buenos Aires. Recuperado de http://www.fundmediterranea.org.ar/images_db/noticias_archivos/3597-345648504.pdf

Pérez Moreno, Elena Silvia (2012). El lugar de las alumnas dentro de una Escuela Técnica Secundaria de la ciudad de Córdoba. Presentado en el 2do. Congreso Interdisciplinario de Género y Sociedad. Córdoba, Universidad Nacional de Córdoba. Recuperado de conferencias.unc.edu.ar/index.php/ponencias/ponencias2013/paper/view/1884/591

Scott, Joan Wallach (1996). El género: una categoría útil para el análisis histórico. En M. Lamas (comp.). El género: la construcción cultural de la diferencia sexual. México: PUEG.

Segato, Rita (2003). Las estructuras elementales de la violencia. Ensayos sobre género entre la antropología, el psicoanálisis y los derechos humanos. Buenos Aires: Editorial Biblos.

Seoane, Viviana. (2017). Diferencia sexual y experiencias de mujeres en la educación técnica: historias de silencios y resistencias. La Aljaba Segunda época, vol. XXI, pp. 29-43. Recuperado de https://cerac.unlpam.edu.ar/index.php/ aljaba/article/view/3343/3234

Seoane, Viviana (2013). Géneros, cuerpos y sexualidades. Experiencias de mujeres en escuelas técnicas de la ciudad de La Plata. Tesis de doctorado. Buenos Aires, Facultad Latinoamericana de Ciencias Sociales. Recuperado de http:// repositorio.flacsoandes.edu.ec/handle/10469/6397

Seoane, Viviana (2012). Sexismo y androcentrismo en la escuela técnica: experiencias de mujeres jóvenes en torno a la sexualidad, el género y la condición juvenil. Presentado en VII Jornadas de Sociología de la Universidad Nacional de La Plata. La Plata. Recuperado de http://www.memoria.fahce.unlp.edu. ar/trab_eventos/ev.2266/ev.2266.pdf 
Silveira, Sara (2011). Género, trabajo y formación profesional: una realidad interdependiente y mutuamente modificante. Género y Trabajo. Avances y Desafíos Pendientes, vol. 4, núm. 11. Recuperado de https://www.oitcinterfor.org/ sites/default/files/file_publicacion/AyA\%2011.pdf

Silveira, Sara (2001). La dimensión de género y sus implicaciones en la relación entre juventud, trabajo y formación. En Enrique Pieck (coord.). Los jóvenes y el trabajo: la educación frente a la exclusión social. México: UIA/IML/Unicef/ Cinterfor/OIT/RET/Conalep. Recuperado de http://www.oitcinterfor.org/ node $/ 7107$

Sobrevila, Marcelo Antonio (1995). La educación técnica argentina. Buenos Aires: Academia Nacional de Educación.

Solari, A. et al. (2012). Transferencia y generación de tecnología para la cadena foresto-industrial. INTA. Recuperado de http://inta.gob.ar/proyectos/corri-430051.

Sosa, Mariana (2018). Inserción laboral de egresados técnicos en dos sectores contrastantes: construcción e informática. En C. Jacinto (coord.). El secundario vale. Saberes, certificados y títulos técnicos en la inserción laboral de jóvenes. Buenos Aires: Miño y Dávila.

Sosa, Mariana (2016). Incidencia de la formación técnica en la inserción laboral juvenil. Los Egresados ETP en el mercado de trabajo en general y en el sector de la construcción en particular en Argentina (2003-2014). Tesis de maestría en Sociología Económica, Instituto de Altos Estudios Sociales, Universidad Nacional de San Martín.

Subirats, Mariana (1986). Niños y niñas en la escuela: una exploración de los códigos de género actuales. En Mariano Enguita (ed.). Marxismo y sociología de la educación. Madrid: Akal.

Sweet, Richard (2006). Los desafíos que enfrenta la educación técnica profesional en Chile: lecciones de la experiencia internacional. Presentado en el III Seminario Internacional DesarrollaT: Trazando el Camino de la Educación Media TP en Chile. Santiago. 